Case Report

\title{
Sarcomatoid Renal Cell Carcinoma Arisen after the Renal Allograft from Donation after Citizen Death: A Case Report and Literature Review
}

\author{
Wu Yonglu,Chen Jie*, Yuan Boxiang, Su Zexuan, Ding Hongwen, Shi Liping, Wang Shaojiang \\ Department of Urology, First Affiliated Hospital of Jinan University, Guangzhou, China \\ Email address: \\ 342126478@qq.com (Chen Jie), 1972109320@qq.com (Wu Yonglu) \\ ${ }^{*}$ Corresponding author
}

To cite this article:

Wu Yonglu, Chen Jie, Yuan Boxiang, Su Zexuan, Ding Hongwen, Shi Liping, Wang Shaojiang. Sarcomatoid Renal Cell Carcinoma Arisen after the Renal Allograft from Donation after Citizen Death: A Case Report and Literature Review. Journal of Surgery.

Vol. 4, No. 5, 2016, pp. 122-125. doi: 10.11648/j.js.20160405.16

Received: August 28, 2016; Accepted: September 12, 2016; Published: October 8, 2016

\begin{abstract}
The aim of this study was to discuss the causes and preventions of sacromatoid renal cell carcinoma (SRCC) after renal allograft from donation after citizens death (DCD). It was reported on a 45 -year-old male patient had undergone renal transplantation in other hospital because of chronic renal insufficiency uraemia in August of 2014. Two months later, lesions found in the renal parenchyma in the right iliac fossa via the color doppler ultrasound. The area of renal transplantation was bulged with tenderness. The renal dysfunction and microscopic haematuria were found. The review color doppler ultrasound and CT showed that the allograft kidney is enlarged rapidly. On $22^{\text {nd }}$ December 2014, a surgical exploration was performed. An irregular and hard mass within the parenchyma of engrafted kidney was found, which was considered as malignant. The nephrectomy of the renal allograft was performed in the operation. First postoperative day, his condition was deteriorating rapidly. Finally, the patient died of cardiac functional failure. The tumor was pathologically diagnosed as sacromatoid renal cell carcinoma (SRCC). SRCC found in the donor allograft kidney is highly malignant and poorly prognosis. The repeated imaging investigations are necessary to exclude the hidden trouble of tumors, such as SRCC. Postoperative immunosuppressive regimens should be modified and the screening program of renal tumor for the early control and prevention should be strengthen.
\end{abstract}

Keywords: Organ Donation, Renal Allograft, Sarcomatoid Renal Cell Carcinoma

\section{Introduction}

Malignancies are one of the main causes of death in kidney transplant recipients. The maligancies had influenced the survival of kidney transplant recipients. The donor-related malignancies is a fatal factor of the organ transplant recipients.It's reported that the donor-related tumor rate is $0.04 \%$ and the overall mortality rate of the patients whose donor related malignancies is $38 \%$ in America [1]. How to prevent the cause of donor-related tumor is a challenge in kidney transplantion domain knowledge. Here was reported a case about the donor-related tumor after kidney transplant. In October 2014, a SRCC patient admitted in our department who had one DCD donor kidney. Sarcomatoid renal cell carcinoma (SRCC) which is high malignancy with poor prognosis rare in clinic. We have retrospective analysis this case with reviewing literature as following.

\section{Case Report}

A 45-year-old male patient admitted to other hospital in August 2014, diagnosed with chronic renal dysfunction (uremic syndrome). He received DCD donor renal allograft transplantation, and also postoperative immunosuppressive regimens - tacrolimus (FK506), mycophenolate mofetil (MMF) and methylprednisolone. The serum concentration of FK506 was regularly monitoring. On $14^{\text {th }}$ October 2014 , the 
follow-up result showed that the concentration of FK506 increased to $19.2 \mathrm{ng} / \mathrm{ml}$ and he was admitted into our department. After the admission, the patient had mild nausea and vomiting and waist pain. He had no fever, frequent micturition, urgent micturition and dysuria. He had no gross hematuria and dysuria. The daily urine volume was $1500-2000 \mathrm{ml}$. For the physical examination, the enlarged and fixed implanted kidney from DCD donor could be palpated with tenderness. The urine routine showed microscopic hematuria. The color doppler ultrasound showed there was a lesion in the renal parenchyma. The resistance index of influx renal artery (IRA 0.73) and segment renal artery (SRA 0.76) were high (Fig 1a.). CT scan noted that there were polycystic masses within the kidney. The structure was not clear although the excretion was functional. It was suggested that the acute renal allograft rejection could occur. The original kidneys were atrophy with polycystic masses. They were non-functional (Fig 2.). The blood routine showed that white blood cell (WBC) was $27.72 \times 10^{9} / \mathrm{L}$, the serum creatinine $(\mathrm{sCr})$ was $284 \mu \mathrm{mol} / \mathrm{L}$. The serum tumor markers and repeated virus detection were negative. After one week, the patient became anuria, the concentration of FK506 decreased to $17.6 \mathrm{ng} / \mathrm{ml}$, and serum creatinine increased to $497 \mu \mathrm{mol} / \mathrm{L}$. The daily urine volume kept to $1700 \mathrm{ml}$ after receiving methylprednisolone and anti-damage treatment. The ultrasound scan in the second week, the size of renal allograft enlarged and the resistance index increased consistently (IRA 0.81 and SRA 0.87). WBC increased to $40.51 \times 10^{9} / \mathrm{L}$ and the neutrophils occupied $90.7 \%$. FK506 was $12.6 \mathrm{ng} / \mathrm{ml}$ and $\mathrm{sCr}$ was still $497 \mu \mathrm{mol} / \mathrm{L}$. Finally, the anti-infectious treatment and hemodialysis were given and the tacrolimus was also adjusted according to the blood concentration of FK506. On $16^{\text {th }}$ December 2014, the ultrasound scan showed that the renal allograft was $88 \mathrm{~mm} \mathrm{x}$ $193 \mathrm{~mm}$ in size which is much bigger than the $10^{\text {th }}$ postoperative day. It was only $57 \mathrm{~mm} \times 106 \mathrm{~mm}$. The structure of the parenchyma was not clear and some hypoechoic mass lesion could be observed. Abdominal CT scan showed that the size of renal allograft increased and it was dysmorphic and there were uneven density (some high density spots and irregular small patches of low density foci which margins were poor defined. Similarly, it suggested the acute renal allograft rejection (Fig.3). The methylprednisolone was given afterwards. However, the WBC climbed to $161.53 \times 10^{9} / \mathrm{L}$, and $\mathrm{sCr}$ was $800 \mu \mathrm{mol} / \mathrm{L}$. The diagnosis was acute renal allograft rejection, but it could not exclude the possibility of tumor. Finally, on $22^{\text {nd }}$ December 2014 a surgery through general anesthesia (GA) was performed. The renal allograft was found injected and bulged. The size of the allograft was $135 \mathrm{~mm} x$ $9.5 \mathrm{~mm} \times 70 \mathrm{~mm}$, on the surface of which multiple nodules were found. The renal artery and vein raised from external iliac artery and vein. The kidney was finally removed. The pathological examination noted that the size of nodules on the surface were $0.5-4.5 \mathrm{~cm}$. The section of the nodules is grey white and yellowish grey. It contained plenty of dead tissues and small amount of poor differentiated cancer cells. Under the light microscope, the cells were heterogenic, presented as spindle-shaped or polygonal. They were multinuclear cells and their nuclei were level 3-4. The cytoplasm contained inclusion body and inflammation cells. The diffuse nodular lesions throughout the kidney. The immunohistochemistry (IHC) results were $\mathrm{CK} 8(+), \mathrm{Vim}(++), \mathrm{AAT}(++)$, EMA focal(+), CK7(-), CK19(-), Desmin(-), SMA(-), myoglobin(-), myosin(-), CEA(-), ki-67 $\sim 40 \%(+)$. It concluded that the results conformed to the SRCC. After $1^{\text {st }}$ postoperative day, the patient exacerbated and died of cardiac functional failure.

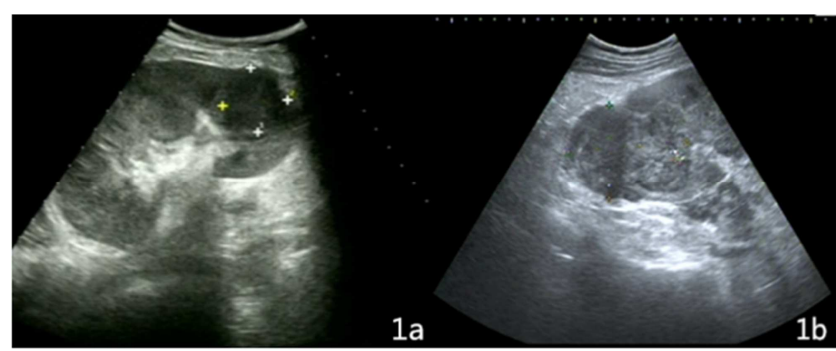

Fig. 1. Ultrasound scan of renal allograft.

1a: (Two months post-operation) Some hypoechoic foci in the parenchyma, the size of larger one: $27 \times 30 \mathrm{~mm}$ with well defined margin.

1b: (Four months post-operation) Structure distortion of renal allograft, some hypoechoic foci in the parenchyma, and the size of the largest one: 58x83mm, extruded on the surface.

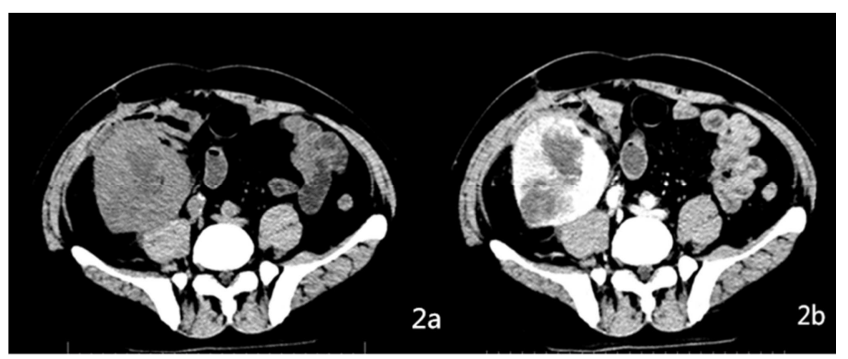

Fig. 2. Abdominal CT scan of renal allograft (two months post-operation).

2a: there are some round hypo-density foci.

2b: For CT contrast, the lesions are dimmer than surroundings normal tissues and poor cortico-medullary differentiation.

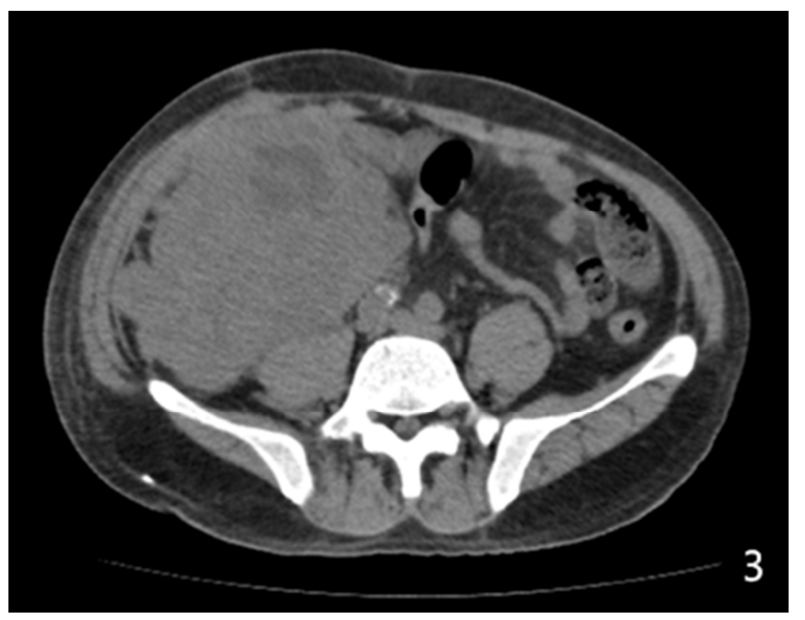

Fig. 3. Abdominal CT scan of renal allograft (four months post-operation).

The size of renal allograft enlarged and it is dysmorphic. There is an irregular hypo-density focus with poor defined margins. 


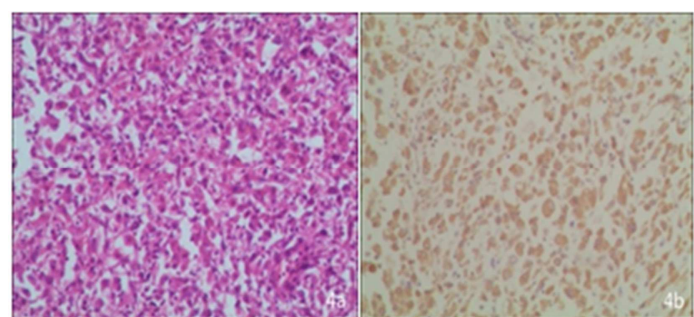

Fig. 4. Pathological results.

4a: Many spindle-like and polyclonal cells (HE x 100), presented as diffuse nodules.

4b: IHC (SP x 100)

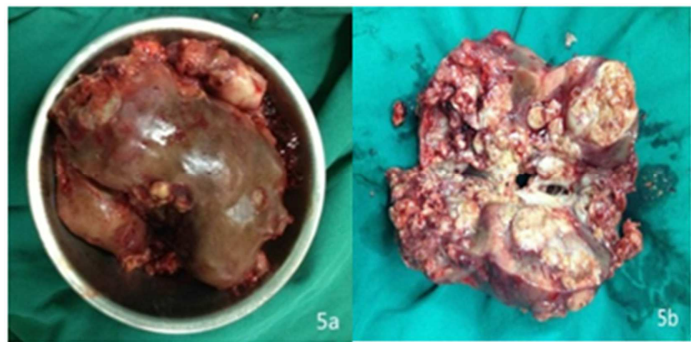

Fig. 5. The resected renal tissues, size: $135 \mathrm{~mm} \times 95 \mathrm{~mm} \times 70 \mathrm{~mm}$, extruded nodules with grey white and yellowish grey cutting surface.

\section{Discussions}

\subsection{The Features of Highly Malignant Tumor After DCD Donor Renal Transplantation}

The survival time is expanding gradually with the modification of transplantation techniques and new developed immunoinhibitors. However, the number of postoperative tumors is increasing as well. The incidence rate of the development of malignant tumors in renal transplant recipients (RTRs) is 3 to 5 times of normal populations, and Some kinds of tumors could even reach 100 times [2]. After 10 postoperative years, the incidence rate would be $15-20 \%$, furthermore, after 20 years, the incidence rate could reach about $40 \%$. Wang Cao et al. reported that a single center had 15120 cases having renal transplantation in 11 years [3]. The incidence rate of malignant tumor is $2.95 \%$, it occupied $55.8 \%$ of tumors of urinary system by recorded. Pedotti et al. [4] also reported that the incidence rate after 10 postoperative years is $4.9 \%$ in China. It is much lower that other countries. It may contribute on using lower dosage of immunoinhibitors and incomplete follow-up system and statistical data. In western countries, skin cancer and lymphatic tumor are much common. On the other hand, the two leading tumors in China are tumors of urinary system and alimentary system [3]. There are several factors to cause these differences between China and western countries, for example, age, gender, geographic environment, lifestyle, primary diseases related factors, and individualized immunosuppressive treatment.

Kidney cancers occupied $2-3 \%$ of malignant cancer in normal population and $80-90 \%$ of malignant cancer located in kidney, especially those aged from 50-70. Tillou et al [5] analyzed 32 centers with 41806 cases having renal allograft transplantation from 1988 to 2012 in France. There are 70 of them diagnosed as new onset tumor of renal allograft. The average time of confirmed diagnosis after transplantation is 142.6 months (11.9years). the average age is 47.5 years $(26.1$ -72.6 years). In 2004, WHO published a classification of types of renal tumors. The most common one is renal clear cell carcinoma $(>75 \%)$, then that are renal papillary carcinoma $(15 \%)$, undifferentiated renal carcinoma (5-10\%) and renal chromophobe cell carcinoma (5\%). Cornelis et al [6] analyzed 11 institutes from 2003 to 2010 , there are 20 cases of de novo renal tumor via laparoscopic treatment. According to its pathological investigations, there are 17 of papillary carcinoma, 3 of clear cell carcinoma and others which are rare types. In this case, it is sacromatoid carcinoma, which belongs to undifferentiated renal carcinoma. It is very rare.

The etiology of malignant tumor of DCD renal allograft is unknown. It is possible that the size of the tumor is undetectable by ultrasound. It also could be caused by viruses, such as EBV, HPV etc. when the patient is immunosuppressed. The viruses turn the tissue into malignancy [7]. In this case, the time to discover the tumor is short. The tumor grows quickly. We could find inclusion bodies and inflammation cells in the pathological tissues. it is probably related to viral infection. The new onset tumor within two years after the operations, we need to think of the donor [8]. The case occurred after 2 postoperative months, it can contribute to the DCD donor.

\subsection{Differentiation Between Renal Carcinoma After Kidney Transplant and Acute Rejection}

Acute rejection after kidney transplant should present as sharply dropping of urine volume, weight increase, temperature increase, discomfort, high blood pressure, enlarge renal allograft with tenderness and dull pain, sCr increase, WBC increase (mainly lymphocyte), proteinuria and hematuria. Color doppler ultrasound showed high vessel resistance. On the other hand, there is a classical triad for renal carcinoma, which includes hematuria, waist pain and abdominal mass. Some of cases will present as bone pain and consistent coughing. The number of asymptomatic renal carcinoma is increasing according to the foreign reports (about 50\%) [9]. Since the symptoms and signs are not classical, both renal carcinoma after kidney transplant and acute rejection could present hematuria, fever, high blood pressure, anemia, swelling of allograft and tenderness. It will affect the diagnosis. In this case, the clinical features are not classical, and only a two-month lesion. All the virus, tumor marker tests are negative. Two ultrasound results show high vessel resistance and CT scan notes that it is acute rejection. The relatives of patient rejected the biopsy for times. In that moment, it was more likely an acute rejection, and then we used MMF and adjust the immunosuppressive drugs to against the rejection. However, the kidney was enlarging and $\mathrm{sCr}$ was increasing until renal failure. Finally, we performed nephrectomy.

It's necessary to evaluate whether the donor kidney has tumor before transplanttion. Because most of the DCD donors need the respirator therapy, it's inconvenient with the donor 
organs undertake MR imaging and CT scan. For this, it can repeated color ultrasound to evalute the donor kidney through different doctors. In addition, the biopsy of pathology also a effective way to evaluate the quality of the donor organs before taking out. Meanwhile, it can performed the biopsy of pathology in different parts of the kidney after the kidney removed form the donor. Expect that, it is about 2\% of deceased donors has a past history of cancer [10]. It should be carefully analysed the clinical history and undertaken a comprehensive clinical examination of each donor. If that, it could reduce the risk of the donor-relatived malignancies.

\subsection{The Prevention and Control of Renal Transplant Tumor}

The malignancy of renal transplant tumor is high and prognosis is very poor. Renal carcinoma occupies $4.6 \%$ of all malignant tumors after transplant[11], but the incidence rate of new onset renal carcinoma of transplant kidney is barely $0.39-0.5 \%$ and the Papillary carcinoma is much common style[6]. SRCC of transplant kidney is a subtype of advanced level transformation. It is not just a pathological subtype [12]. It is clinically rare, especially in China. It is highly invasive and metastatic. It could affect surrounding tissues of kidney and some common distant metastatic sites, such as bones, lungs, liver and brain. It often belongs to III or IV stage when we discover it. Therefore, the prognosis is very poor $[13,14]$. The tissue morphology of SRCC is similar to renal sarcoma carcinosarcoma. It needs pathological biopsy to differentiate it. Generally, SRCC is composed of carcinoma tissue and sarcoma-like tissue, they could transit with each other and tightly connected. It has a feature of epithelial differentiation. It means that it may arise from renal tubule epithelia [14]. It reported that renal cell carcinoma has two high expressions Cytokeratin and Vimentin, about 52\%-100\%. It notes that it can differentiate into two ways [15].

After the transplantation, if tumor is discovered, the immunosuppression should be stopped. It is because it could probably cause acute rejection. SRCC is not sensitive to chemotherapy, but only surgical procedure. For that local renal tumor which radius is $<4 \mathrm{~cm}$, could reserve the part of kidney [5]. There are some articles mention that thermal ablation is effective to SRCC and also lower the mortality [6]. If the tumor is widely distributed, or uncontrolled acute rejection, we have to perform radical nephrectomy. Luo Ming et al [16] reported that Sirolimus could be used as non-surgical option.

\section{Conclusion}

Before transplant, DCD organs should be checked by imaging examinations for times, such as color duplex ultrasound and CT scan for screening. The donor and receiver are required to do virus and tumor marker tests before the operation. Urinary cytology is necessary as well. We should exclude the potential tumor strictly. After the operation, we need to modify the immunosuppressive treatment and do the screening test for new onset tumor. It could enhance the curative effect of organ transplant, if we discover and treat as early as possible.

\section{References}

[1] Kauffman H M, McBride M A, Cherikh W S, et al. Transplant tumor registry: donor related malignancies. Transplantation, 2002, 74 (3): 358-362.

[2] Hwang JK, Moon IS, Kim JI. Malignancies after kidney transplantation: a 40-year single-center experience in Korea. Transpl Int 2011; 24: 716-21.

[3] Wang Chao, Li Tao, Zhang Jian, Liu Fen, Xie Zelin, Tang Yawang, et al. Trend analysis of malignant tumor after renal transplantation in China. Organ Transplantation 2015; 3: 169-73.

[4] Pedotti P, Cardillo M, Rossini G, Arcuri V, Boschiero L, Caldara $\mathrm{R}$, et al. Incidence of cancer after kidney transplant: results from the North Italy transplant program. Transplantation 2003; 76: 1448-51.

[5] Tillou X, Guleryuz K, Doerfler A, Bensadoun H, Chambade D, Codas R, et al. Nephron sparing surgery for De Novo kidney graft tumor: results from a multicenter national study. American Journal of Transplantation 2014; 14: 2120-5.

[6] Cornelis F, Buy X, André M, Oyen R, Bouffard-Vercelli J, Blandino A, et al. De novo renal tumors arising in kidney transplants: midterm outcome after percutaneous thermal ablation. Radiology 2011; 260: 900-7.

[7] Schulz TF. Cancer and viral infections in immunocompromised individuals. International journal of cancer 2009; 125: 1755-63.

[8] Nalesnik MA, Ison MG. Organ transplantation from deceased donors with cancer: is it safe? Open Access Surgery 2011; 4: 11-20.

[9] Wein AJ, Kavoussi LR,Novick AC,Partin AW, Peters CA. Renal Tumors.In: Guo Yinglu, Zhou Liqun, editor. Campbell-Walsh Urology, $9^{\text {th }}$ ed. Peking: Peking University Medical Press; 2009.p.1651-773.

[10] Kauffman H M, Cherikh W S, McBride M A, et al. Deceased donors with a past history of malignancy: an organ procurement and transplantation network/united network for organ sharing update. Transplantation, 2007, 84 (2): 272-274.

[11] Penn I. Primary kidney tumors before and after renal transplantation.T ransplantation, 1995, 59 (4): 480-485.

[12] De Peralta-Venturina M, Moch H, Amin M, Tamboli P, Hailemariam S, Mihatsch M, et al. Sarcomatoid differentiation in renal cell carcinoma: a study of 101 cases. The American journal of surgical pathology 2001; 25: 275-84.

[13] Ro JY, Ayala AG, Sella A, Samuels ML, Swanson DA. Sarcomatoid renal cell carcinoma: clinicopathologic. A study of 42 cases. Cancer 1987; 59: 516-26.

[14] Akhtar M, Tulbah A, Kardar A H, Ali MA. Sarcomatoid renal cell carcinoma: the chromophobe connection. The American journal of surgical pathology 1997; 21: 1188-95.

[15] Xu Hongmin, Li Weihua, Li Hongfen. The expression of cytokeratin and vimentin immunohistochemistry in renal cell carcinoma. Medical Journal of Chinese People's Liberation Army 1988; 3:177-9.

[16] Luo Ming, Qiu Jian-xin. Mammalian target of rapamycin inhibitors in the treatment of malignancy after organ transplantation. World clinical drugs 2009:02:65-71. 